\title{
A non-linear second-order stochastic model of ocean surface waves
}

\author{
Maminirina JOELSON*, Alfred RAMAMONJIARISOA
}

Institut de recherche sur les phénomènes hors équilibre, laboratoire IOA, case 903, Parc scientifique et technologique de Luminy, 13288 Marseille cedex 9, France

\begin{abstract}
This paper deals first with the mathematical formulation of a non-linear second-order stochastic model of free irrotational ocean surface wave on deep water. Then, the case of wave motion of zero bandwidth is treated to illustrate the model. On the basis of the usual hydrodynamic equations, the formulation is made by successive applications of the harmonic decomposition and the so-called Wiener-Hermite functional series expansion of random functions. This procedure yields the kernels equations. These kernels allow to obtain the random surface wave fields from the Wiener set of elementary random processes. For the particular case cited above, as expected, the second-order non-linearity results in the generation of a second harmonic component of the fundamental component. The corresponding realisations of the random variations of the water surface deflection level exhibit close similarity with data from laboratory experiments. The potentiality of the approach for modelling a wide class of random oceanic processes is stressed. (C) 2001 Ifremer/CNRS/IRD/Éditions scientifiques et médicales Elsevier SAS
\end{abstract}

Résumé - Modèle non linéaire du second ordre d'ondes de surfaces océaniques. Cet article concerne en premier lieu la formulation mathématique d'un modèle non linéaire, du second ordre, d'ondes de surface océaniques aléatoires, sur profondeur infinie. Ensuite, le cas d'un champ d'ondes de largeur de bande nulle est traité pour illustrer la potentialité du modèle. Sur la base des équations usuelles de l'hydrodynamique, la formulation est effectuée en faisant appel successivement à la décomposition harmonique puis aux développement fonctionnel en série dit de "Wiener-Hermite » des fonctions aléatoires. Cette procédure conduit aux équations aux noyaux. Ceux-ci caractérisent la transformation qui permet d'obtenir les champs d'ondes de surface à partir de l'ensemble des processus aléatoires élémentaires de Wiener-Hermite. Pour le cas particulier cité précédemment, on constate que la non linéarité conduit à la génération d'une composante harmonique du deuxième ordre de l'onde fondamentale. Les échantillons de variations du niveau de la surface libre, construits d'après le modèle, montrent des similitudes frappantes avec les échantillons issus d'expériences en laboratoire. L'intérêt de la méthode pour modéliser une large classe de phénomènes océaniques aléatoires est mis en relief. (C) 2001 Ifremer/CNRS/IRD/Éditions scientifiques et médicales Elsevier SAS

Fourier-Stiltjes decomposition / kernels equations / oceanic surface waves / random functions / Wiener-Hermite functional series

représentation de Fourier-Stieltjes / équations aux noyaux / ondes de surface océaniques / fonctions aléatoires / série fonctionnelle de Wiener-Hermite

\section{INTRODUCTION}

The development of theoretical stochastic models of ocean surface wave is known as a topic of considerable

*Correspondence and reprints: fax: +33491419620. interest from both the fundamental and the practical viewpoints. However, the progress on this topic is strikingly much smaller than that made on deterministic fields. In the past, extensive use of the so-called harmonic decomposition have been made, the main objective being the determination of the wave field spectrum. The literature on water surface waves refers to a considerable work 
devoted to this quantity. However, the models so developed cannot be classified as stochastic models in the strict sense. Indeed, a stochastic model requires as main result the multidimensional probability density functions of the physical variables of interest Sobczyk. 1984). The determination of these functions from the basic hydrodynamic equations is known to be extremely difficult and no real attempt seems to exist at this time in that respect.

One other approach consists in finding random solutions to the equations. These are solutions of stochastic nonlinear boundary value problem. Unfortunately, the mathematical foundations for such problems seems to be not yet clearly established.

From the theoretical viewpoint, a valuable approach consists in the determination of realisations of the random physical quantities. In that respect, the use of the socalled Wiener-Hermite functional series expansion appears quite promising. This method has been applied with significant success in the field of turbulence in fluid flows Meecham and Siegel, 1964; Meecham and Jeng, 1968. Meecham and Clever, 1971 and in the field of stochastic non-linear dynamical systems Jahedi and Ahmadi 1983]. If desired, second-order statistical properties can be determined, but, in addition, others properties can be specified from the realisations.

A first application of the method to potential water surface wave on deep water has been carried out by the authors (Joelson and Ramamonjiarisoa, 1999) with encouraging results. The obtained wave field is Gaussian. Herein, further application is made by introducing second-order non-linear effect which, in the spirit of the Wiener-Hermite expansion, would yield to a small departure from a Gaussian field. Such departure is often observed in experimental results.

In section 2, the basic hydrodynamic equations are recalled. Transformation in the Fourier space is made to yield to the specification of the second-order non-linear model. Projection in the Wiener-Hermite space follows with derivation of the kernels equations whose solutions are determined. In section 3, the specific example of zero bandwidth fundamental wave motion is treated and the results briefly discussed by comparison with experimental data. The main conclusions are reported in the last section.

\section{MATHEMATICAL FORMULATION OF THE PROBLEM}

\subsection{The basic hydrodynamic equation and boundary conditions}

The water surface wave field of interest is assumed to be governed by the usual set of equation and boundary conditions for irrotational motion on deep water (see e.g. Phillips, 1977)

Let $t, x, y$, and $z$ be respectively the time, the horizontal coordinates and the vertical coordinate; $g, \varphi$ and $\eta$, the gravity acceleration, the velocity potential and the water surface elevation; the set of interest writes:

Laplace equation:

$$
\varphi_{x x}+\varphi_{y y}+\varphi_{z z}=0
$$

Kinematic boundary condition:

$$
\eta_{t}+\varphi_{x} \eta_{x}+\varphi_{y} \eta_{y}-\varphi_{z}=0 \quad \text { at } \quad z=\eta
$$

Dynamic boundary condition:

$$
g \eta+\varphi_{t}+\frac{1}{2}\left(\varphi_{x}^{2}+\varphi_{y}^{2}+\varphi_{z}^{2}\right)=0 \text { at } z=\eta
$$

Radiation condition:

$$
\lim _{z \rightarrow-\infty} \varphi=0
$$

\subsection{Wiener-Hermite functional series expansion and kernels equations}

Details on the Wiener-Hermite functional expansion can now be found in textbooks and papers (see e.g. Wiener 1958: Meecham and Siegel, 1964: Jahedi and Ahmadi, 1983:-Joelson and Ramamonjiarisoa, 1999). We briefly recall that the expansion constitutes a mathematical procedure which allows to represent an arbitrary random function in terms of an ensemble of elementary random processes, namely, the Wiener set (Wiener, 1958). This particular nature of the elementary components is to be stressed with respect to many usual expansions procedures. 
More precisely, given an arbitrary random function $f(t)$ of $t$ (time), with zero mean, $f(t)$ can be expanded in the following functional form:

$$
\begin{gathered}
f(t)=\int_{-\infty}^{+\infty} F_{1}\left(t, t_{1}\right) H^{(1)}\left(t_{1}\right) \mathrm{d} t_{1}+ \\
\int_{-\infty}^{+\infty} \int_{-\infty}^{+\infty} F_{2}\left(t, t_{1}, t_{2}\right) H^{(2)}\left(t_{1}, t_{2}\right) \mathrm{d} t_{1} \mathrm{~d} t_{2}+\ldots \\
\int_{-\infty}^{+\infty} \int_{-\infty}^{+\infty} \ldots \int_{-\infty}^{+\infty} F_{j}\left(t, t_{1}, \ldots, t_{j}\right) H^{(j)}\left(t_{1}, t_{2}, \ldots, t_{j}\right) \mathrm{d} t_{1} \mathrm{~d} t_{2} \ldots \mathrm{d} t_{j}+
\end{gathered}
$$

where $H^{(1)}, H^{(2)}, \ldots, H^{(j)}, \ldots$ are respectively the first, second, ..., $j^{\text {th }}, \ldots$ members of the Wiener set while $F_{1}, F_{2}, \ldots, F_{j}, \ldots$ are respectively the first, second, $\ldots, j^{\text {th }}, \ldots$ order kernels.

The first order member, $H^{(1)}$, of the Wiener set is the so-called Gaussian 'white noise' process with zero mean and $\delta$-correlated. The others members are defined by combinations of first-order processes (see e.g. Meecham and Siegel.1964). Of fundamental importance is that the kernels are deterministic functions of the arguments. They represent mathematically the projection of the random function $f(t)$ on the Wiener set. Clearly, if the expansion is limited to the first term, $f(t)$ will be Gaussian. Then, the higher order terms represent the departure from a normal process.

In the important particular case of a stationary random function, it may appear convenient to apply the expansion in the Fourier rather than in the physical space, through the usual Fourier-Stieltjes transformation. In particular, this may greatly help in interpreting the results on physical grounds. We have:

$$
f(t)=\int_{\omega} \mathrm{d} A(\omega) \exp \mathrm{i} \omega t
$$

To this decomposition corresponds the following expansion of the (complex) random amplitude $\mathrm{d} A(\omega)$ :

$$
\begin{aligned}
& \mathrm{d} A(\omega)=A_{1}(\omega) \bar{H}^{(1)}(\omega)+ \\
& (2 \pi) \int_{\omega_{1}} A_{2}\left(\omega_{1}, \omega-\omega_{1}\right) \bar{H}^{(2)}\left(\omega_{1}, \omega-\omega_{1}\right) \mathrm{d} \omega_{1}+\ldots
\end{aligned}
$$

Again, the kernels $A_{1}, A_{2}, \ldots$ are deterministic functions of the arguments. They are the Fourier transformation of $F_{1}, F_{2}, \ldots$, while $\bar{H}^{(1)}, \bar{H}^{(2)}, \ldots$ are the Fourier-Stieltjes transformation of $H^{(1)}, H^{(2)}, \ldots, H^{(j)}, \ldots$. Note that the set $\bar{H}^{(j)}$ keeps the orthogonality property of the original set $H^{(j)}$ (see e.g. Loelson. 1997. More generally, for a random function, homogeneous in the horizontal plane (x) and stationary in time, the classical harmonic decomposition allows first to write:

$$
f(\mathbf{x}, t)=\int_{\mathbf{k}} \int_{\omega} d A(\mathbf{k}, \omega) \exp i(\mathbf{k} \cdot \mathbf{x}-\omega t)
$$

then, the Wiener-Hermite expansion of the amplitude is:

$$
d A(\mathbf{k}, \omega)=A_{1}(\mathbf{k}, \omega) \bar{H}^{(1)}(\mathbf{k}, \omega)+
$$

$$
\begin{array}{r}
(2 \pi)^{3} \int_{\mathbf{p}} \int_{q_{1}} A_{2}(\mathbf{p}, q, \mathbf{k}-\mathbf{p}, \omega-q) \bar{H}^{(2)} \\
(\mathbf{p}, \omega, \mathbf{k}-\mathbf{p}, \omega-q) \mathrm{d} \mathbf{p} \mathrm{d} q
\end{array}
$$

Under the above assumptions, the physical variables of interest can be written as:

$$
\begin{array}{r}
\eta(\mathbf{x}, t)=\int_{\mathbf{k}} \int_{\omega} d N(\mathbf{k}, \omega) \exp i(\mathbf{k} \cdot \mathbf{x}-\omega t) \\
\varphi(\mathbf{x}, z, t)=\int_{\mathbf{k}} \int_{\omega} \exp (k z) d B(\mathbf{k}, \omega) \exp i(\mathbf{k} \cdot \mathbf{x}-\omega t)
\end{array}
$$

Through lengthy and tedious algebra whose detail can be found in Leelson (1997), the general expressions of the boundary conditions in the Fourier space can be written (Joelson, 1999). Note that the procedure used is similar to that by Phillips (1960) who looked at the case of an homogeneous but non-stationary wave field. These general expressions involve infinite sums of terms. If truncated at order $n$, the sums will define model at order $(n+1)$.

\subsection{The second-order model}

According to the above, by limiting the sums to their respective first terms, a second-order model is defined. It is characterised by the following boundary conditions: 
- kinematic condition:

$$
\begin{gathered}
i \omega d N(\mathbf{k}, \omega)+k d B(\mathbf{k}, \omega)= \\
-\iint_{\mathbf{k}, \omega}\left[\left|\mathbf{k}-\mathbf{k}^{\prime}\right|^{2}+\mathbf{k}^{\prime} \cdot\left(\mathbf{k}-\mathbf{k}^{\prime}\right)\right] d N\left(\mathbf{k}^{\prime}, \omega^{\prime}\right) \\
d B\left(\mathbf{k}-\mathbf{k}^{\prime}, \omega-\omega^{\prime}\right) d \mathbf{k}^{\prime} d \omega^{\prime}
\end{gathered}
$$

- dynamic condition:

$$
\begin{gathered}
g d N(\mathbf{k}, \omega)=i \omega d B(\mathbf{k}, \omega)= \\
i \iint_{\mathbf{k}, \omega}\left(\omega-\omega^{\prime}\right)\left|\mathbf{k}-\mathbf{k}^{\prime}\right| d N\left(\mathbf{k}^{\prime}, \omega^{\prime}\right) d B \\
\left(\mathbf{k}-\mathbf{k}^{\prime}, \omega-\omega^{\prime}\right) d \mathbf{k}^{\prime} d \omega^{\prime}- \\
\frac{1}{2} \iint_{\mathbf{k}, \omega}\left[k^{\prime}\left|\mathbf{k}-\mathbf{k}^{\prime}\right|-\mathbf{k}^{\prime} \cdot\left(\mathbf{k}-\mathbf{k}^{\prime}\right)\right] d B\left(\mathbf{k}^{\prime}, \omega\right) d B \\
\left(\mathbf{k}-\mathbf{k}^{\prime}, \omega-\omega^{\prime}\right) d \mathbf{k}^{\prime} d \omega^{\prime}
\end{gathered}
$$

To derive the kernels equations in the Wiener-Hermite expansions, the random amplitudes $\mathrm{d} N(\mathbf{k}, \omega)$ and $\mathrm{d} B(\mathbf{k}$, $\omega)$ are first written according to the development in equation (9). Then, the developments are introduced in the above boundary conditions. Finally a quite complicated and lengthy algebra (for detail, see Joelson. 1997) yields the following equations:

$$
\begin{aligned}
& i \omega \eta^{(1)}(\mathbf{k}, \omega)+k \varphi^{(1)}(\mathbf{k}, \omega)=0 \\
& g \eta^{(1)}(\mathbf{k}, \omega)-i \omega \varphi^{(1)}(\mathbf{k}, \omega)=0
\end{aligned}
$$

$i\left(\omega_{1}+\omega_{2}\right) \eta^{(2)}\left(\mathbf{k}_{1}, \omega_{1}, \mathbf{k}_{2}, \omega_{2}\right)+$

$$
\left|\mathbf{k}_{1}+\mathbf{k}_{2}\right| \varphi^{(2)}\left(\mathbf{k}_{1}, \omega_{1}, \mathbf{k}_{2}, \omega_{2}\right)=
$$

$\left(\frac{1}{2 \pi}\right)^{3}\left(\mathbf{k}_{1}+\mathbf{k}_{2}\right)^{2}\left[\eta^{(1)}\left(\mathbf{k}_{1}, \omega_{1}\right) \varphi^{(1)}\left(\mathbf{k}_{2}, \omega_{2}\right)\right]$

and

$$
\begin{aligned}
& g \eta^{(2)}\left(\mathbf{k}_{1}, \omega_{1}, \mathbf{k}_{2}, \omega_{2}\right)- \\
& i\left(\omega_{1}+\omega_{2}\right) \varphi^{(2)}\left(\mathbf{k}_{1}, \omega_{1}, \mathbf{k}_{2}, \omega_{2}\right)=\left(\frac{1}{2 \pi}\right)^{3} \times \\
& {\left[-i\left(\omega_{1} k_{1}+\omega_{2} k_{2}\right) \varphi^{(1)}\left(\mathbf{k}_{1}, \omega_{1}\right) \eta^{(1)}\left(\mathbf{k}_{2}, \omega_{2}\right)+\right.} \\
& \left.\frac{1}{2}\left(k_{1} k_{2}-\mathbf{k}_{1} \cdot \mathbf{k}_{2}\right)\right] \times \varphi^{(1)}\left(\mathbf{k}_{1}, \omega_{1}\right) \varphi^{(1)}\left(\mathbf{k}_{2}, \omega_{2}\right)
\end{aligned}
$$

where $\eta^{(1)}$ and $\varphi^{(1)}$ are the first-order kernels while $\eta^{(2)}$ and $\varphi^{(2)}$ are the second-order kernels and $k_{i}=\left|\mathbf{k}_{i}\right|$ $(i=1,2)$.

\subsection{Solutions}

Equations (14) and (15) yield immediately to a dispersion relation and a relationship between the two firstorder kernels, namely:

$$
\omega^{2}=g k
$$

and

$$
\varphi^{(1)}=-i \frac{\omega}{k} \eta^{(1)}
$$

Then, equations (16) and (17) allow to write the secondorder kernels in terms of the first-order kernels. For the application which will follow, we will be interested in the water surface elevation random variation. The corresponding second-order kernel is written:

$$
\begin{gathered}
\eta^{(2)}\left(\mathbf{k}_{1}, \omega_{1}, \mathbf{k}_{2}, \omega_{2}\right)= \\
\left(\frac{1}{2 \pi}\right)^{3}\left[\frac{\left(\omega_{1}+\omega_{2}\right) F \frac{\omega_{2}}{k_{2}}-K \frac{\omega_{1}}{k_{1}}\left(G+\frac{1}{2} H \frac{\omega_{2}}{k_{2}}\right)}{\left(\omega_{1}+\omega_{2}\right)^{2}-g K}\right] \times \\
\eta^{(1)}\left(\mathbf{k}_{1}, \omega_{1}\right) \eta^{(1)}\left(\mathbf{k}_{2}, \omega_{2}\right)
\end{gathered}
$$

in which $\quad F=\left(\mathbf{k}_{1}+\mathbf{k}_{2}\right)^{2}, \quad G=\left(\omega_{1} k_{1}+\omega_{2} k_{2}\right)$, $H=k_{1} k_{2}-\mathbf{k}_{1} \cdot \mathbf{k}_{2}$ and $K=\left|\mathbf{k}_{1}+\mathbf{k}_{2}\right|$.

\section{ILLUSTRATIVE EXAMPLE}

Let us consider the specific case where the first-order kernels correspond to the Dirac delta function, namely:

$$
\begin{aligned}
\eta^{(1)}\left(\mathbf{k}_{i}, \omega_{i}\right)=\frac{A_{0}}{2}\left[\delta\left(\mathbf{k}_{i}-\mathbf{k}_{0}, \omega_{i}-\omega_{0}\right)+\right. \\
\left.\delta\left(\mathbf{k}_{i}+\mathbf{k}_{0}, \omega_{i}+\omega_{0}\right)\right]
\end{aligned}
$$

with $i=1,2 . A_{0}$ and $\mathbf{k}_{0}$ are constant amplitude and wave number and the notation $\delta(\mathbf{k}, \omega)=\delta(\mathbf{k}) \delta(\omega)$ is used for simplicity. 
Taking into account equations (9) and (20), the expression of the random amplitude of the water surface deflection level is now of the form:

$$
\begin{aligned}
& d N(\mathbf{k}, \omega)=\frac{A_{0}}{2}\left[\delta\left(\mathbf{k}-\mathbf{k}_{0}, \omega-\omega_{0}\right)+\right. \\
& \left.\delta\left(\mathbf{k}+\mathbf{k}_{0}, \omega+\omega_{0}\right)\right] \bar{H}^{(1)}(\mathbf{k}, \omega)+ \\
& \frac{A_{0}^{2}}{4} \int_{\mathbf{p}} \int_{q_{1}} M(\mathbf{p}, q, \mathbf{k}-\mathbf{p}, \omega-q) \\
& {\left[\begin{array}{l}
\delta\left(\mathbf{p}-\mathbf{k}_{0}, q-\omega_{0}\right) \delta\left(\mathbf{k}-\mathbf{p}-\mathbf{k}_{0}, \omega-q-\omega_{0}\right)+ \\
\delta\left(\mathbf{p}+\mathbf{k}_{0}, q+\omega_{0}\right) \delta\left(\mathbf{k}-\mathbf{p}+\mathbf{k}_{0}, \omega-q+\omega_{0}\right)
\end{array}\right] \times} \\
& \bar{H}^{(2)}(\mathbf{p}, \omega, \mathbf{k}-\mathbf{p}, \omega-q) d \mathbf{p} d q
\end{aligned}
$$

which immediately gives:

$$
\begin{aligned}
& d N(\mathbf{k}, \omega)=\frac{A_{0}}{2}\left[\delta\left(\mathbf{k}-\mathbf{k}_{0}, \omega-\omega_{0}\right)+\right. \\
& \left.\delta\left(\mathbf{k}+\mathbf{k}_{0}, \omega+\omega_{0}\right)\right] \bar{H}^{(1)}(\mathbf{k}, \omega)+ \\
& \frac{A_{0}^{2}}{4}\left[M\left(\mathbf{k}_{0}, \omega_{0}, \mathbf{k}-\mathbf{k}_{0}, \omega-\omega_{0}\right)\right. \\
& \delta\left(\mathbf{k}-2 \mathbf{k}_{0}, \omega-2 \omega_{0}\right) \times \\
& \bar{H}^{(2)}\left(\mathbf{k}_{0}, \omega_{0}, \mathbf{k}-\mathbf{k}_{0}, \omega-\omega_{0}\right)+ \\
& M\left(-\mathbf{k}_{0},-\omega_{0}, \mathbf{k}+\mathbf{k}_{0}, \omega+\omega_{0}\right) \delta\left(\mathbf{k}+2 \mathbf{k}_{0}, 2 \omega_{0}\right) \times \\
& {\left[\bar{H}^{(2)}\left(-\mathbf{k}_{0},-\omega_{0}, \mathbf{k}+\mathbf{k}_{0}, \omega+\omega_{0}\right)\right]}
\end{aligned}
$$

From equation (23), the variations comprises two components: a first-order Gaussian component, $\eta_{1}(\mathbf{x}, t)$, and a second-order, non-Gaussian component, $\eta_{2}(\mathbf{x}, t)$.

$$
\eta(\mathbf{x}, t)=\eta_{1}(\mathbf{x}, t)+\eta_{2}(\mathbf{x}, t)
$$

Clearly, the second-order component is at wavenumber and frequency $2 \mathbf{k}_{0}$ and $2 \omega_{0}$ respectively. Then, the second-order non-linear effect results in the generation of the second harmonic component of the primary wave.

A simple algebra allows to show that:

$$
\begin{array}{r}
\eta_{1}(\mathbf{x}, t)=A_{0} \int_{\mathbf{x}_{1}} \int_{t_{1}} \cos \left[\left(\mathbf{x}-\mathbf{x}_{1}\right) \cdot \mathbf{k}_{0}+\left(t-t_{1}\right) \omega_{0}\right] \\
H^{(1)}\left(\mathbf{x}_{1}, t_{1}\right) d \mathbf{x}_{1} d t_{1}
\end{array}
$$

and

$$
\begin{array}{r}
\eta_{2}(\mathbf{x}, t)=\frac{A_{0}^{2}}{2} M_{0} \int_{\mathbf{x}_{1}} \int_{t_{1}} \int_{\mathbf{x}_{2}} \int_{t_{2}} \cos \left[\left(\mathbf{x}-\mathbf{x}_{1}+\mathbf{x}-\mathbf{x}_{2}\right) \cdot \mathbf{k}_{0}\right. \\
\left.+\left(t-t_{1}+t-t_{2}\right) \omega_{0}\right] \times \\
H^{(2)}\left(\mathbf{x}_{1}, t_{1}, \mathbf{x}_{2}, t_{2}\right) d \mathbf{x}_{1} d t_{1} d \mathbf{x}_{2} d t_{2}
\end{array}
$$

where $M_{0}$ corresponds to the expression in the square brackets in equation (20) in which $k_{1}$ and $k_{2}$ are replaced by $k_{0}$ while $\omega_{1}$ and $\omega_{2}$ are replaced by $\omega_{0}$. We have:

$$
M_{0}\left(k_{0}, \omega_{0}\right)=\frac{2 k_{0} \omega_{0}^{2}}{2 \omega_{0}^{2}-g k_{0}}
$$

with

$$
\omega_{0}^{2}=g k_{0}
$$

Equations (25) and (26) allow to construct realisations of $\eta(\mathbf{x}, t)$ although such construction is not a simple matter because its involves computations of multiple convolution integrals.

We will illustrate the random wave forms by considering the simplified, only time-dependent, equations:

$$
\eta_{1}(t)=A_{0} \int_{t_{1}} \cos \left[\left(t-t_{1}\right) \omega_{0}\right] H^{(1)}\left(t_{1}\right) d t_{1}
$$

and

$$
\begin{array}{r}
\eta_{2}(t)=\frac{A_{0}^{2}}{2} M_{0} \int_{t_{1}} \int_{t_{2}} \cos \left[\left(t-t_{1}+t-t_{2}\right) \omega_{0}\right] \\
H^{(2)}\left(t_{1}, t_{2}\right) d t_{1} d t_{2}
\end{array}
$$

Eigure displays samples of $H^{(1)}(t)$ and then $\eta_{1}(t)$ according to equation (29) while Goure 2 displays samples of $H^{(2)}\left(t_{1}, t_{2}\right)$ and then $\eta_{2}(t)$ according to equation (30).

Finally, figure 3 displays detailed aspects of $\eta_{1}(t), \eta_{2}(t)$ and $\eta(t)=\eta_{1}(t)+\eta_{2}(t)$.

The remarkable fact, from figure 3, is that, with respect to the first-order wave form, the second-order component makes the wave crests sharper and the wave trough flatter. This coincides with the second-order effect in deterministic waves (Stokes waves) and is generally observed in experiments in laboratory facilities on wind-generated waves. 

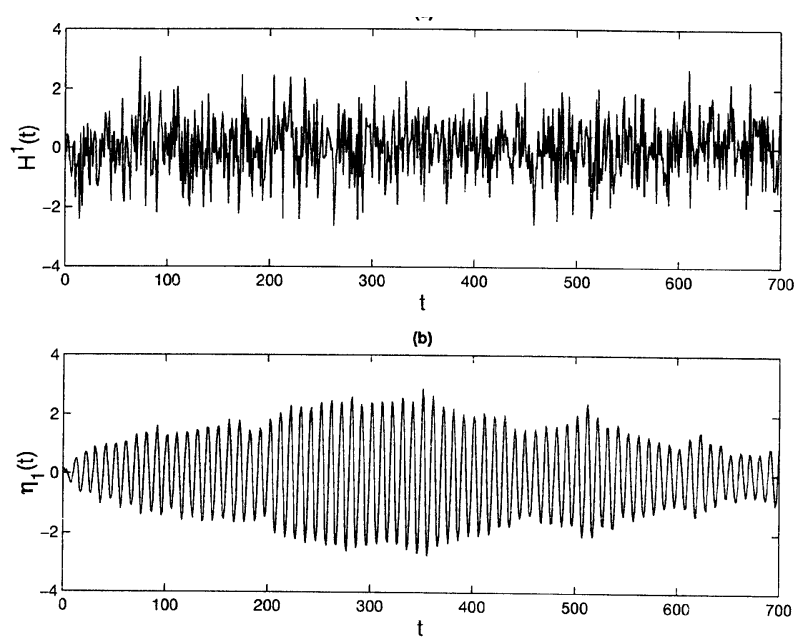

Figures 1. (a) Sample function of the Wiener process $H^{(1)}(t)$; (b) realisation of the first order component $\eta_{1}(t)$ (in arbitrary units) of the random variation of the water surface deflection level (see equation (29)).

\section{CONCLUSION}

This article deals mainly with the mathematical formulation of a non-linear second-order model of stochastic ocean surface waves. The key aspect of the formulation is the combination of the usual Fourier decomposition with the so-called Wiener-Hermite functional series expansion of random functions. The analysis yields the equations for
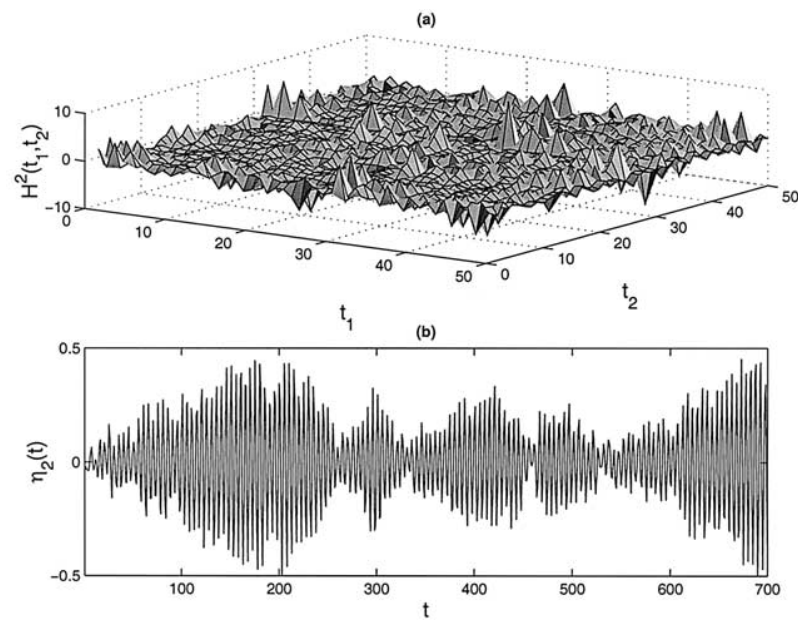

Figures 2. (a) Sample function of the Wiener process $H^{(2)}\left(t_{1}, t_{2}\right)$; (b) realisation of the second-order component $\eta_{2}(t)$ (in arbitrary units) of the random variation of the water surface deflection level (see equation (30)).

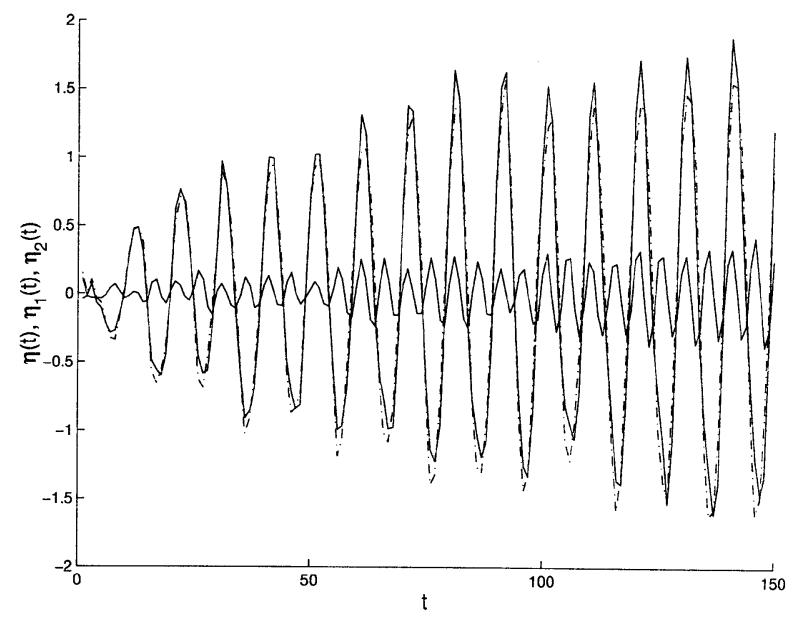

Figure 3. Detailed aspects of $\eta_{1}(t)(\cdot-), \eta_{2}(t)(--)$ and $\eta(t)(-)$ (in arbitrary units).

the kernels which characterise the transformation from the Wiener set of elementary random processes to the surface wave fields. The particular case of a zero bandwidth first-order spectrum is treated as an important illustrative example. It is seen that the second-order non-linearity results, as expected, in the generation of the second harmonic component of the fundamental wave. The corresponding sample function (limited to the time domain) of the water surface deflection level exhibits striking similarity with wave forms observed in laboratory experiments. Namely, with respect to the primary wave profile, the second-order non-linearity results in sharper wave crests and flatter wave troughs.

Subsequent work will be addressed to the construction of realisations in the time-space domain and to the determination of the ratio between the energy levels of the fundamental and the second harmonic components.

Combined with previous investigations in various fields, this work brings in complementary proof of the potentiality of the Wiener-Hermite expansion. The method would greatly help in analysing various random oceanic motions in which stochasticity cannot be ignored (internal waves, turbulence, ...). Unfortunately, as already pointed out by Joelson (1999), severe limitations in practical uses of the method may come first from the algebraic complexity to derive the kernel equations from the basic hydrodynamic equations and, second, to solve these equations which constitute generally coupled, nonlinear, integral or integro-differential equations. The 
symbolic calculus developed by Imamura_et_al_(1965 may be of great help.

\section{REFERENCES}

Imamura, T., Meecham, W.C., Siegel, A., 1965. Symbolic calculus of the Wiener process and Wiener-Hermite functionals. J. Math. Phys. 6, 695-703.

Jahedi, A., Ahmadi, G., 1983. Application of Wiener-Hermite expansion to non stationary vibration of a Duffing oscillator. J. Appl. Mech. 50, 436-442.

Joelson, M., 1997. Contribution à la caractérisation et à la formulation mathématique d'ondes de surface océaniques, Ph.D. thesis. université de la Méditerranée, Aix-Marseille II.

Joelson, M., Ramamonjiarisoa, A.A., 1999. Functional serie expansion method for modelling random fields of water surface waves. In: Banner, M.L. (Ed.), The Wind-Driven Air-Sea Interface. Electro- magnetic and Acoustic Sensing, Wave Dynamics and Turbulence Fluxes. The Univ. of New South Wales, Sydney, Australia, pp. 133-140.

Meecham, W.C., Clever, W.C., 1971. Use of Cameron-Martin-Wiener representations for non linear random processes applications, Proc. Symp. on Statistical Models and Turbulence. Univ. of California, San Diego, pp. 205-229.

Meecham, W.C., Jeng, D.T., 1968. Use of the Wiener-Hermite expansion for nearly normal turbulence. J. Fluid Mech. 32, 225-249.

Meecham, W.C., Siegel, A., 1964. Wiener-Hermite expansion in model turbulence at large Reynolds numbers. Phys. Fluids 7, 1578-1590.

Phillips, O.M., 1960. On the dynamics of unsteady gravity waves of finite amplitude Part 1. J. Fluid Mech. 9, 193-217.

Phillips, O.M., 1977. The Dynamics of The Upper Ocean. Cambridge Univ. Press, Cambridge.

Sobczyk, K., 1984. Stochastic Wave Propagation. PWN-Polish Scientific Publishers, Warszawa.

Wiener, N., 1958. Non Linear Problems in Random Theory. MIT Press. 